\title{
Estratégias de marketing: Estudo no setor de agências de viagens e turismo
}

\author{
Marketing strategies: Study in travel agencies and tourism sector
}

\section{Estrategias de marketing: Estudio en el sector de agencias de viajes y turismo}

\author{
Aléssio Bessa Sarquis ${ }^{1}$ \\ Nádia Pizzinatto \\ Antônio Carlos Giuliani ${ }^{3}$ \\ Andréa Simone Machiavelli Pontes ${ }^{4}$
}

\begin{abstract}
Resumo: $O$ setor de turismo tem papel econômico-social relevante e as estratégias de marketing podem contribuir para melhorar o desempenho de mercado das organizações de serviços. Assim, o estudo analisou as estratégias de marketing aplicadas no setor de serviços de agências de viagens/turismo no Estado de Santa Catarina e, para isso, uma pesquisa quantitativa, tipo descritiva não conclusiva, na forma de levantamento de campo (survey) e de corte transversal foi aplicada com questionários autoadministrados disponibilizados na plataforma Google Docs para uma amostra de 109 agências emissivas de pequeno e médio portes. As análises estatísticas foram realizadas por meio de moda, frequência, média aritmética, desvio-padrão e teste de hipóteses, conforme as escalas de mensuração aplicadas. Os resultados apontam que a grande maioria das agências pesquisadas utiliza estratégias de marketing interno, externo, interativo e de gestão do relacionamento com clientes e que as agências de porte médio utilizam com maior frequência a maioria das estratégias de marketing mensuradas.
\end{abstract}

Palavras-chave: Estratégia de marketing. Relacionamento com clientes. Organizações de serviços. Agências de viagens e turismo.

Abstract: The tourism sector has an important economic and social role and marketing strategies can help improve the market performance of service organizations. Thus, the study analyzed the marketing strategies applied in the travel agency services sector in the state of Santa Catarina and, therefore, a quantitative, descriptive inconclusive and in the form of field survey (survey) was applied with self-administered questionnaires available in the Google Docs platform for a sample of 109 emissive agencies of small and medium. Statistical analyzes were performed by means, frequency, standard deviation and hypothesis testing as the measurement scales applied. The results show that the

1 Doutor em Administração pela Universidade de São Paulo e professor do Programa de Pós-Graduação Mestrado em Administração da Universidade do Sul de Santa Catarina (UNISUL), Florianópolis - Santa Catarina - Brasil. Email: alessio.sarquis@unisul.br

2 Doutora em administração pela Escola de Administração de Empresas de São Paulo da Fundação Getúlio Vargas (EAESP/FGV) e professora do Programa de Pós-Graduação Doutorado em Administração da Universidade Metodista de Piracicaba, Piracicaba - São Paulo - Brasil. E-mail: nkp@nadiamarketing.com.br

3 Doutor em educação pela Universidade Metodista de Piracicaba (UNIMEP) e coordenador do Programa de PósGraduação Mestrado e Doutorado em Administração da Universidade Metodista de Piracicaba, Piracicaba - São Paulo - Brasil. E-mail: cgiuliani@unimep.br

4 Mestre em administração pela Universidade do Sul de Santa Catarina (UNISUL) e professora pela Faculdade de Tecnologia do Senac Caçador, Caçador - Santa Catarina - Brasil. E-mail: andrea.pontes@sc.senac.br 
vast majority of the surveyed agencies use internal marketing strategies, external, interactive and relationship management with clients and midsize agencies use more often most measured marketing strategies.

Keywords: Marketing strategy. Relationship with customers. Service organizations. Travel agencies and tourism.

Resumen: El sector del turismo tiene un papel económico y social importante y estrategias de marketing puede ayudar a mejorar el funcionamiento del mercado de las organizaciones de servicios. Así, el estudio analiza las estrategias de marketing aplicadas en el sector de los servicios de agencias de viajes/turismo en el Estado de Santa Catarina y, por tanto, una cuantitativa, descriptiva poco concluyente y en forma de estudio de campo (encuesta) se aplicó con cuestionarios autoadministrados disponibles en la plataforma de Google Docs para una muestra de 109 agencias emisoras de pequeñas y medianas empresas. Los análisis estadísticos se realizaron por medio de la moda, la frecuencia, media aritmética, desviación estándar y la prueba de hipótesis como las escalas de valoración aplicados. Los resultados muestran que la gran mayoría de las agencias encuestadas utilizan estrategias de marketing internos, gestión interactiva y relación externa con los clientes y las agencias medianas utilizan con más frecuencia las estrategias de marketing medidos.

Palabras clave: Estrategia de mercadeo. Relación con los clientes. Las organizaciones de servicios. Agencias de viajes y turismo.

\section{INTRODUÇÃO}

O setor de turismo é constituído por organizações de diferentes portes e ramos de atividade, e desempenha um papel econômico-social relevante na economia nacional. Em 2011, segundo a Organização Mundial de Turismo, a quantidade de turistas internacionais atingiu no Brasil um nível recorde (nove milhões de visitantes) e o volume de gastos dos turistas cresceu 14,4\%, atingindo US\$ 6,7 milhões (OMT, 2010). Estudo realizado pela Fundação Instituto de Pesquisas Econômicas (Ministério do Turismo, 2014) revelou que em 2011 a quantidade de brasileiros que viajaram pelo país atingiu 58,9 milhões de pessoas; em 2007 o país teve 49,7 milhões de brasileiros viajantes. Esse crescimento foi explicado pela inclusão do turismo de baixa renda, que cresceu $21 \%$ no período.

Dentre as organizações que atuam no setor de turismo, as agências de viagens/turismo se destacam pela quantidade de empresas e pelo papel de intermediação desempenhado no processo de comercialização dos serviços de turismo (Kotler, Bowen, \& Makens, 2006; Kavoura et al., 2013). Conforme Beni (2006), as agências de viagens/turismo são organizações que prestam intermediação comercial e serviços de viagens e turismo. Oferecem, por exemplo, serviços de transporte, hotelaria, atrações turísticas e organizam viagens, e apoiam programas turísticos desenvolvidos por outras organizações (ex: operadores turísticos) e pelos próprios consumidores. Tradicionalmente, essas organizações desempenham um papel relevante no setor, pois atuam no canal de distribuição do setor, prestando serviços especializados, produzindo pacotes turísticos e estimulando a demanda no setor (Borges \& Guardia, 2012; Navickas \& Malakauskaite, 2015).

Segundo Standing, Tang-Taye, e Boyer (2014), com o advento da Internet, diversas mudanças ocorreram no comportamento dos consumidores e fornecedores de serviços turísticos, inclusive no ramo de agências de viagens/turismo. Os consumidores passaram a pesquisar mais, a montar seus próprios roteiros de viagem e a contratar sozinhos passagens aéreas, hotéis, passeios e acompanhamento de guias turísticos, por exemplo. Enquanto os fornecedores do setor (ex: ope- 
radores turísticos, hotéis e companhias aéreas) passaram a vender pela Internet diretamente aos consumidores. Até então, tais serviços eram realizados predominantemente pelas agências de viagens/turismo, que possuem contratos firmados com operadores turísticos e demais fornecedores do setor. Essas mudanças forçaram as agências de viagens/turismo a intensificar seus investimentos em marketing/vendas, inclusive pelo uso de ferramentas da tecnologia de informação e comunicação (Da Silva Flores, De Sena Cavalcante, \& Raye, 2012).

No Brasil, a pesquisa de Barros (2005) apontou que as agências de viagens/turismo estão pressionadas pela redução do comissionamento das companhias aéreas (as agências costumam receber comissão pela venda de passagens), pelo surgimento de concorrentes virtuais (lojas online que operam com maior escala e estrutura de custo enxuta), e pela ampla disponibilidade de informações na Internet sobre destinos e produtos turísticos. Os resultados do estudo apontam também que as agências de viagens/turismo não estão inovando na intensidade necessária, nem explorando suficientemente as potencialidades da Internet, e que há necessidade de ampliar os estudos acadêmicos sobre estratégias de marketing aplicadas no setor.

Estudo de Polo-Peña, Frías-Jamilena e Rodríguez-Molina (2012) apontou que estratégias de marketing são essenciais no ramo de agências de viagens/turismo, em decorrência da evolução do mercado e de mudanças no comportamento dos clientes, principalmente pelo advento das novas tecnologias/Internet. Queiroga (2011) apontou que as estratégias de marketing no setor de turismo são influenciadas por fatores externos, incluindo questões políticas, econômicas, sociais e tecnológicas, e que a disponibilidade de informações, inovações, profissionalização e qualidade nos serviços contribui para a obtenção de vantagem competitiva. A pesquisa de Pelissari et al. (2011), que analisou as estratégias de marketing em uma organização de serviços, revelou que as estratégias não são essencialmente elaboradas conforme a teoria, nem bem formuladas, havendo falta de sintonia em relação aos objetivos da organização. O estudo bibliométrico de Tomazzoni e Bock (2013) analisou a produção científica sobre marketing e gestão estratégica publicada nos principais periódicos acadêmicos de turismo do Brasil e revelou que a maioria dos estudos se concentra em analisar os atributos valorizados pelos clientes e que há necessidade de investigar melhor tópicos como qualidade, inovação, relacionamento com clientes e aplicação de novas tecnologias de gestão. Freire (2005) apontou também que no Brasil mais pesquisas são necessárias sobre estratégias de marketing em agências de viagens/turismo.

O estudo de Borges e Guardia (2012) analisou a produção acadêmica sobre agências de viagens/turismo publicada em revistas Qualis/CAPES ligadas ao setor de turismo, e revelou a existência de poucos estudos acadêmicos sobre o assunto e apenas 18 estudos foram encontrados, principalmente pesquisas exploratórias e qualitativas. O estudo bibliométrico de Rocha e Silva (2006) analisou a produção científica sobre marketing de serviços no Brasil e identificou que a maioria dos estudos aborda o comportamento de compra e os construtos qualidade e satisfação em serviços. Nos estudos identificados, há clara preferência pela perspectiva hipotético-dedutiva e pelos métodos quantitativos de análise, com testes empíricos e uso de instrumentos de medida desenvolvidos por pesquisados de outros países. Os autores sugerem que estudos sobre a prática 
de marketing de serviços em organizações de diferentes regiões e culturas precisam ainda ser realizados.

Consulta realizada no portal (sitio) das universidades que possuem cursos de graduação ou pós-graduação em turismo no Estado de Santa Catarina (UNIVALI - Universidade do Vale do Itajaí, UNISUL - Universidade do Sul de Santa Catarina, FURB - Universidade Regional de Blumenau, IFSC Instituto Federal de Santa Catarina e IES/SC - Instituto de Ensino Superior de SC) revelou que há carência de estudos sobre agências de viagens/turismo na região, especialmente sobre a prática de estratégias de marketing e relacionamento com clientes.

Com base no contexto apresentado, decidiu-se pela realização do presente estudo com o seguinte problema de pesquisa: quais as estratégias de marketing aplicadas pelas organizações de serviços do setor de agências de viagens/turismo do Estado de Santa Catarina? Assim, o objetivo geral foi identificar as estratégias de marketing aplicadas pelas organizações do ramo de agências de viagens/turismo do Estado de Santa Catarina. Para tanto, um estudo descritivo estatístico foi realizado com representantes de 109 agências de turismo de pequeno e médio portes do Estado de Santa Catarina.

\section{ESTRATÉGIAS DE MARKETING PARA SERVIÇOS DE TURISMO}

Segundo a American Marketing Association (AMA, 2014), marketing é uma função organizacional e um conjunto de processos que envolvem a criação, a comunicação e a entrega de valor aos clientes, bem como a administração do relacionamento com clientes, de modo que beneficie a organização e seu público-alvo. Embora o marketing de serviços tenha diferenças na praxis em relação ao marketing de produtos manufaturados, a sua definição não carece de enunciado diferente e os princípios básicos que fundamentam o marketing são igualmente aplicáveis para bens, serviços e organizações (Kotler et al., 2014; Sarquis, 2009). No entanto, a definição de serviços é complexa e variada, já que o serviço é intangível, gera entendimento nebuloso e compreende um conjunto de atividades de natureza pessoal, empresarial e/ou governamental. No contexto deste estudo, serviço compreende um "ato, atividade, ação, esforço, atuação, desempenho ou processo, normalmente intangível, que é oferecido à venda, na forma de um conjunto de benefícios, sem resultar na propriedade de algo, com o objetivo de satisfazer necessidades, e que envolve algum tipo de interação entre o prestador de serviço e o cliente" (Sarquis, 2009, p.2).

As organizações de serviços necessitam de estratégias de marketing para obter o desempenho de mercado desejado, conforme Cavalcanti (2008) e Ghemawat e Rivkin (2006), e estas podem estar relacionadas à escolha de mercados-alvo, lançamento de produtos, prospecção de novos clientes e ao relacionamento com clientes, por exemplo. Conforme Sarquis e Ikeda (2009), estratégias de marketing são meios empregados pela organização para a consecução dos objetivos de marketing estabelecidos e podem incluir decisões sobre a aplicação de recursos e sobre a implementação dos esforços mercadológicos da organização. Já para Kotler et al. (2014), estratégias de marketing são meios pelos quais a organização se relaciona com o mercado, e incluem aspectos 
relacionados à decisão sobre produtos, preços, promoções, prazos e relacionamento com os clientes.

Nas organizações de serviços de turismo, as estratégias de marketing devem ser definidas a partir da avaliação dos objetivos de mercado da organização, características dos clientes-alvo, forma de atuação da concorrência e demais variáveis relevantes do ambiente (Kotler, Bowen, \& Makens, 2006). O desempenho das estratégias de marketing depende do conhecimento da organização sobre produtos e mercados, e do planejamento adequado da matriz de produto-mercado (Hollensen, 2014). Quando executado de forma adequada, o planejamento das estratégias de marketing possibilita direcionar melhor a organização para mercados atraentes, estabelecer vantagens competitivas, melhorar a comunicação interna e otimizar a alocação dos recursos de marketing da organização (Sarquis \& Pizzinatto, 2013).

As estratégias de marketing para serviços de turismo podem incluir também aspectos relacionados à segmentação de mercado, seleção de públicos-alvo, diferenciação competitiva, posicionamento de marca e definições sobre produtos, preços, distribuição, logística, comunicação e vendas da organização. Segundo Lovelock, Patterson e Wirtz (2014), o composto de marketing para serviços é constituído por um conjunto de recursos e ferramentas que podem ser aplicados na definição das estratégias de marketing da organização e auxiliar na tarefa de alcançar os objetivos de mercado. No caso do mix de comunicação/vendas, exemplos de estratégias de marketing aplicadas no setor de turismo são investir em propaganda ou promoção de vendas, promover eventos patrocinados e buscar negócios em novos mercados. No caso do mix de processos de serviços, exemplos são elevar os padrões de qualidade dos serviços, melhorar a satisfação dos clientes e elevar a produtividade da organização em relação aos concorrentes.

Nos serviços de turismo, as estratégias de marketing podem também estar relacionadas à introdução de inovações em produtos, processos organizacionais e na forma de relacionamento com clientes (Kotler, Bowen, \& Makens, 2006; Da Silva Flores, De Sena Cavalcante, \& Raye, 2012). O mercado de turismo exige cada vez mais qualidade e menor custo, e o nível de competição tem se intensificado ao longo do tempo (Machado et al, 2011; Scarpin, Mondini, Neumann, \& Machado, 2011). Conforme Barboza et al. (2013), a introdução de inovações é necessária em mercados com concorrência acirrada, variedade de ofertas, mensagens promocionais frequentes e elevado nível de exigência dos consumidores.

No âmbito dos serviços, as estratégias de marketing têm sido classificadas em externo, interno e interativo (Grönroos, 1984; Sarquis, 2009). O marketing externo compreende aquelas estratégias de marketing tradicionais, desenvolvidas por profissionais especialistas e orientadas para o mercado-alvo da organização. Já o marketing interno envolve o conjunto de estratégias relacionadas à preparação dos recursos internos (sistemas, gestores e funcionários) para a implementação de programas e promessas de marketing externo da organização. Enquanto, o marketing interativo compreende estratégias direcionadas aos processos de produção e consumo de serviços da organização, aquelas geralmente implementadas por profissionais não especializados em marketing, tais como clientes e funcionários de linha de frente da organização (front office). Sobre o as- 
sunto, o estudo de Huang e Rundle-Thiele (2014) revelou que no setor de turismo a cultura tem efeito moderador da prática de marketing da organização e do relacionamento interno com os funcionários.

De maneira geral, as organizações de serviços de turismo empregam estratégias de marketing no relacionamento com clientes, seja na prospecção de novos negócios ou na prestação de serviços de pós-venda. Como a demanda de serviços é diversificada, sazonal e requer a participação dos clientes, aspectos do perfil dos clientes e da forma de interação nos serviços são também estratégias de marketing relevantes no setor (Sarquis \& Ikeda, 2009; Polo-Peña, Frías-Jamilena, \& Rodríguez-Molina, 2012). Outra premissas do marketing de serviços de turismo é o foco na satisfação dos clientes e no desenvolvimento de relacionamentos de longa duração. Nesse sentido, algumas estratégias de marketing indicadas são (Sampaio \& Fagundes, 2008; Dwyer \& Schurr, 2006): conhecer os consumidores-alvo, superar as expectativas dos clientes, ofertar serviços com agilidade, ofertar serviços personalizados, desenvolver relacionamento pessoal com os clientes, promover facilidades de acesso, ofertar promoções periódicas, dispor de funcionários competentes, dispor de atendimento cordial e executar ações de pós-venda. O estudo de Kavoura et al. (2013) no setor de turismo revelou a necessidade de cooperação com os demais agentes de mercado na implementação das estratégias de marketing, e que a incorporação de tecnologias de informação, ações de comunicação e c-commerce podem fortalecer as redes/alianças com mercado e apoiar na promoção/comercialização de destinos turísticos.

No Brasil, o estudo de Barboza et al (2013) analisou a gestão de marketing em organizações de serviços e revelou que $92 \%$ das organizações pesquisadas utilizam alguma estratégia do composto promocional, e que produto e preço são estratégias de diferenciação importantes, e que as estratégias de marketing podem contribuir para melhorar o desempenho de mercado da organização. Vasconcelos, Souza e Guimarães (2010) analisaram uma organização de serviços com elevada satisfação dos clientes e qualidade de serviços, e concluiu que as estratégias são relevantes e podem contribuem para o diferencial competitivo da organização. Sarquis e Ikeda (2009) investigaram a prática de segmentação de mercado e seleção de mercados-alvo em organizações de serviços e revelaram que a maioria das organizações estudadas adota estratégias de segmentação de mercado, mas carece de sistemáticas de aplicação mais estruturadas e de maior esforço na implementação; que a totalidade das organizações pesquisadas adota a estratégia de marketing diferenciado, e costuma identificar novos mercados e classificar os clientes em segmentos, mesmo que de maneira informal.

Já Okada e Souza (2011) analisaram as publicações sobre estratégias de marketing para serviços e identificaram que inovações tecnológicas criaram tendências de mercado e causam impacto na gestão de marketing das organizações, incluindo novas demandas dos consumidores e decisões de compra baseadas em características intrínsecas do produto, e que as facilidades dos mecanismos de buscas e o aumento de consumo exigem estratégias de marketing mais inovadoras e diferenciadas. Já Lucena (2011) analisou o processo de formulação de estratégias de marketing em uma organização de serviços, especificamente o processo de formação de estratégias, o con- 
texto onde as estratégias foram desenvolvidas, as estratégias aplicadas e as mudanças ocorridas ao longo do tempo. Os resultados apontaram que as principais estratégias de marketing aplicadas estão ligadas ao produto e à comunicação de marketing, foram implantadas com propósito de ganhar market share, atender determinadas necessidade dos clientes, diversificar a abordagem de mercado e obter vantagem competitiva; que outras estratégias de marketing utilizadas são posicionamento de marca e aspectos de precificação, distribuição, segmentação e diferenciação competitiva.

O estudo de Ribeiro, Grisi e Saliby (1999) analisou a aplicação do marketing de relacionamento no mercado de serviços e os resultados apontam benefícios tanto para a organização como para os clientes. Para a organização, o marketing de relacionamento permite acesso direto ao mercado, conhecimento mais profundo sobre os clientes e melhor qualidade no atendimento. Para os clientes, a estratégia permite obter maior satisfação, facilidade na procura de alternativas e maximização na troca econômica. Já a pesquisa de Santa Maria (2009) analisou estratégias de marketing de relacionamento aplicadas nas relações com stakeholders em 28 organizações no setor de serviços e os resultados indicam que estas estratégias são aplicadas de forma não sistemática, sem planejamento e controle dos resultados, e que isso afeta o desempenho da organização; também identificou que as estratégias de marketing mais utilizadas são campanhas publicitárias e relações públicas; e que há necessidade de melhorar a implementação das estratégias de comunicação, principalmente a alimentação do sitio institucional, a edição de revistas/periódicos, a promoção de encontros/eventos, as atividades da ouvidoria, e a participação dos funcionários no relacionamento com clientes.

Rocha et al. (2013) investigaram a utilização de redes sociais na construção do relacionamentos com clientes por meio de estudo de múltiplos casos. Os resultados indicam que as organizações de serviços utilizam redes sociais para oferecer conteúdo e para a prestação de serviços aos clientes; que há nas organizações pesquisadas oportunidades de aprimoramento dos serviços on-line, e que a oferta de novos serviços pode contribuir para melhorar o relacionamento e a qualidade percebida dos serviços. Já o estudo de Carvalho, Hemais e Motta (2001) analisou o comportamento dos consumidores em ambientes de serviços com uso de músicas e os resultados apontam que poucos consumidores perceberam espontaneamente a musicalidade no ambiente, que a música é mais percebida quando é desagradável ou ajuda a construir uma experiência positiva. No entanto, consumidores e funcionários avaliam que a música contribui para a criação de um ambiente propício à produção e entrega de serviço.

Santos e Fernandes (2008) examinaram a recuperação de serviços sob a perspectiva de ferramenta de relacionamento com clientes. Os autores fizeram proposta e teste de um modelo teórico com foco no inter-relacionamento entre avaliações específicas do processo de reclamação, confiança, custo de mudança, valor e lealdade do consumidor. Os resultados indicam que a confiança e lealdade do consumidor são afetadas pela forma como as reclamações são resolvidas; que as percepções de justiça afetam a satisfação com o processo de reclamação; que a confiança do consumidor é influenciada pela satisfação no pós-reclamação; que tanto a intenção de recompra 
como a comunicação boca-a-boca são influenciadas pela confiança, satisfação e valor percebido. $O$ estudo aponta também que o gerenciamento das reclamações tem papel fundamental na construção de relacionamentos com clientes, podendo ser também elemento de diferenciação competitiva.

No contexto do setor de turismo, o estudo de Freire (2005) analisou o uso de estratégias de marketing nos serviços de hotelaria e revelou que qualidade e inovação são estratégias relevantes, e que o desconhecimento sobre estratégias de marketing é ainda um grande entrave na prática das organizações. $O$ estudo revelou também que os dirigentes pesquisados conhecem apenas por intuição as necessidades dos clientes, e que as organizações investem mais em estratégias de divulgação da marca, principalmente em propaganda, promoção de vendas, relações públicas, marketing direto, marketing on-line e comunicação boca a boca. Maske (2012) estudou a relação entre orientação empreendedora, orientação de mercado, grau de inovação e desempenho organizacional em 70 empresas do setor de turismo, e os resultados apontam que os gestores pesquisados procuram ativamente por inovações e avaliam que a inovação tem papel importante no desempenho da organização, seguido da orientação empreendedora e da orientação para o mercado. Sobre o assunto, Queiroga (2011) avaliou o processo de formação de estratégias de marketing em empreendimentos de serviços e revelou que as estratégias de marketing são influenciadas por fatores do ambiente externo, como tendências políticas, econômicas, sociais e tecnológicas; que as principais estratégias de marketing utilizadas são segmentação de mercado, posicionamento de mercado, diferenciação competitiva, e aspectos de produto, precificação, distribuição e comunicação de marketing.

No ramo de agências de viagens/turismo, Da Silva Flores, De Sena Cavalcante e Raye (2012) estudaram o uso da tecnologia da informação e comunicação no relacionamento com clientes, fornecedores/parceiros, na comercialização de serviços e nas estratégias de marketing, e os resultados indicam que é ainda reduzido a quantidade de agências que possuem site próprio, que o email marketing é utilizado com intensidade equivalente ao telefone, e que as mídias sociais têm sido bastante utilizadas, principalmente no relacionamento com clientes. Radu et al. (2014) analisaram o impacto do marketing on-line e concluíram que, na última década, com o advento da Internet, a quantidade de agências de viagens/turismo off-line (aquela com instalação física de ponto de venda) caiu mais de $50 \%$, e que cada vez mais os consumidores estão obtendo informações sobre serviços turísticos por intermédio de boca-a-boca on-line, portais on-line e web/sites corporativos. Assim, as agências de viagens/turismo precisam adaptar suas estratégias utilizando mais marketing de mídia social, aplicativos móveis, marketing de relacionamento e serviços diferenciados.

Rodríguez et al. (2014) analisaram o uso de programas de afiliação como estratégia de marketing no contexto de agências de viagens/turismo, por meio de pesquisa qualitativa e entrevistas em profundidade com expert e profissionais do setor. O estudo revelou que a aplicação de programas de afiliação está se desenvolvendo gradualmente nas organizações; que é percebido como menos importante pelas agências off-line; que as estratégias mais utilizadas são páginas da Web com conteúdo específico e de interesse pessoal, sites que distribuem receitas de vendas com os usuários 
finais, bancos de dados de e-mail marketing e a construção de redes de afiliados. Sobre esse assunto, Cid et al. (2015) analisaram a influência das redes sociais no ramo de agências de viagens/turismo e revelaram que, com o avanço da Internet, estratégias tradicionais de marketing estão sendo substituídas por estratégias contemporâneas, baseadas na tecnologia da informação e comunicação, e que os fornecedores do setor começaram a criar canais de marketing direto com os consumidores buscando melhorar a eficiência dos serviços e reduzir os custos de comercialização. Esse fenômeno tem forçado as agências de viagens/turismo a se adaptarem ao mercado e a investirem mais em estratégias de marketing, principalmente o uso de redes sociais.

Viljoen, Roberts-Lombard e Jooste (2015) desenvolveram um modelo teórico de estratégias de marketing para reintermediação de serviços no contexto das agências de viagens/turismo, a partir de discussões de grupos focais e uma pesquisa online com profissionais do setor. Segundo o modelo proposto, as estratégias de marketing focadas nos clientes-alvo (ex: interação pessoal, serviço diferenciado, redução nas taxas de serviço, conhecimento do produto, serviço de alta qualidade e uso de tecnologia) contribuem para reduzir os efeitos da desintermediação no setor de turismo e para elevar a retenção dos clientes. Para isso, as agências precisam investir na reconquista dos clientes perdidos, ampliar o mix de produtos e oferecer serviços diferenciados e mais inovações em marketing.

Hoffmann et al. (2015) analisaram as estratégias de marketing adotadas no setor hoteleiro, visando identificar aquelas que melhor contribuem para o desempenho da organização, por meio de estudo quantitativo, na forma de levantamento de campo (survey) e questionário em 23 hotéis brasileiros. Os resultados apontam como principais estratégias a reputação da marca, a cultura organizacional, a oferta de espaços para eventos, a localização do hotel e a qualidade dos serviços, incluindo o padrão dos quartos e alimentos. Os resultados contém também evidências de que uma estratégia importante é desenvolver relações com outras organizações que participam da cadeia produtiva do turismo, mesmo que esta não tenha revelado qualquer associação com outras variáveis como cooperação, confiança e intercâmbio de informações.

Por fim, o estudo de Moyano et al. (2015) analisou o desenvolvimento de estratégias de marketing com base na satisfação do consumidor em eventos, por meio de questionário aplicado com 488 turistas. Os resultados apontam que na elaboração da estratégia de marketing a perspectiva do cliente deve ser considerada (ex: percepções sobre o evento e avaliações da satisfação), e que as estratégias de marketing podem ser agrupadas em quatro categorias: vendas, comunicação sem interação, oferta de benefícios e comunicação com Interação. As estratégias relacionadas com vendas são aquelas que buscam estimular a compra e a recompra pelos clientes. As de comunicação sem Interação dedicam-se a manter os consumidores informados sobre o evento e estimular indiretamente o processo de vendas. As estratégias de oferta de benefícios procuram dar ao cliente um prêmio pelo histórico de relacionamento e/ou pelo desempenho no evento. Ao passo que, a comunicação com interação envolve as estratégias que buscam estabelecer um canal de comunicação direto com o consumidor, principalmente com propósito de fomentar a correta utilização dos serviços. 


\section{ASPECTOS METODOLÓGICOS}

O presente estudo envolveu a aplicação de pesquisa quantitativa, descritiva não conclusiva, na forma de levantamento de campo (survey) e de corte transversal. Conforme Richardson (2008), a pesquisa quantitativa caracteriza-se pelo emprego de quantificação na amostragem, no método de coleta e na análise dos dados, enquanto a pesquisa descritiva é aquela que busca descrever as características de grupos, estimar proporção de elementos ou descobrir a existência de relação entre variáveis (Collins; Hussey, 2005). O presente estudo teve propósito não conclusivo por empregar um processo de amostragem não probabilístico e com seleção não aleatória dos elementos amostrais.

A população do estudo compreende as agências de viagens/turismo emissivas instaladas no Estado de Santa Catarina registradas no Ministério do Turismo do Brasil na data-base 30/março/2014, de pequeno e médio portes. A listagem das 226 agências de viagens/turismo identificadas foi elaborada a partir do mailing list de dois importantes fornecedores do setor (Operadora Central de Intercâmbio e New Line Tour Operator), escolhidos em função do tempo de existência (mais de 20 anos), credibilidade de mercado e abrangência de atuação no Estado de Santa Catarina. As agências de viagens/turismo emissivas foram selecionadas porque mais estruturadas, possuem um mix de serviços mais amplo e maior possibilidade de investimento em estratégias de marketing/vendas (Candioto, 2012). O tamanho da amostra resultou em 109 organizações. As fontes de informações foram proprietários, gestores e/ou colaboradores com amplo conhecimento na organização pesquisada.

A coleta de dados envolveu a aplicação de questionário autoadministrado, disponibilizado na plataforma Google Docs e apoiado por carta-convite personalizada, enviado ao universo das agências de viagens/turismo do Estado de Santa Catarina identificadas. O instrumento de coleta compreendeu um questionário estruturado com 61 perguntas em formato fechado, sendo 07 sobre perfil das agências de viagens/turismo, 12 sobre estratégias de marketing externo, 10 sobre marketing interno e interativo, 11 sobre estratégias de relacionamento com clientes e 21 sobre as estratégias de marketing que mais contribuem para vantagem competitiva. As perguntas foram elaboradas em formato fechado e em escalas de 5 pontos (onde 1="discordo totalmente", $5=$ "concordo totalmente" e $0="$ não sabe") e baseadas nos objetivos do estudo e no referencial teórico. No instrumento de coleta, as questões sobre estratégias de marketing externo foram elaboradas a partir dos estudos de Freire (2005) e Polo-Peña, Frías-Jamilena e Rodríguez-Molina (2012); sobre marketing interno e interativo nos estudos de Huang e Rundle-Thiele (2014) e Standing, Tang-Taye e Boyer (2014); sobre relacionamento com clientes nos trabalhos de Kotler, Bowen e Makens (2006) e Sarquis (2009).

$\mathrm{Na}$ análise dos dados, os questionários foram inicialmente conferidos na verificação do preenchimento, e depois na análise da consistência interna pela técnica Alpha de Cronbach (Cronbach, 1951), sendo que o resultado para o conjunto de variáveis foi 0,906 e, quando analisado por variável, este valor ficou sempre entre 0,903 e 0,906, atendendo o nível de confiabilidade elevada segundo Murphy e Davidsholfer (1988). Os dados foram também tabulados e analisados com auxí- 
lio do SPSS (Statistical Package for the Social Sciences) versão 22, conforme as escalas de mensuração do questionário aplicado. As perguntas com escala nominal foram analisadas por meio de moda e distribuição de frequência relativa (medidas de dispersão); enquanto as perguntas com escala intervalar e razão foram analisadas por meio de média aritmética (medida de tendência central), distribuição de frequência e desvio-padrão estimado (medidas de dispersão).

As agências de viagens/turismo pesquisadas foram também analisadas quanto ao porte pela quantidade de funcionários. Foi classificada como agência de pequeno porte aquela que dispunha de até 9 funcionários e como médio porte aquela com 10 a 99 funcionários. Essa classificação foi elaborada a partir do modelo proposto pelo SEBRAE (Serviço Brasileiro de Apoio às Micro e Pequenas Empresas) para organizações de comércio e serviços, adaptado para atender aos propósitos do presente estudo. $O$ teste t-Student para amostras independentes (não pareadas) foi também aplicado para verificar se há diferencias estatísticas entre opiniões dos pesquisados em função do porte/tamanho da organização.

\section{APRESENTAÇÃO E ANÁLISE DOS RESULTADOS}

Quanto ao perfil das agências de viagens/turismo pesquisadas, os dados coletados indicam que a grande maioria delas ( $78 \%$ da amostra) é constituída por organizações que existem há mais de 6 anos, que atuam em todo o Estado de Santa Catarina (67\%) e em outros estados do Brasil (57\%), que atendem a clientes pessoa física (90\%) e empresas privadas (79\%) e a consumidores das classes sociais B e C (85\%) e classe A (72\%). Do total das agências pesquisadas, $82,6 \%$ são organizações de pequeno porte e $17,4 \%$ organizações de médio porte, sendo que as agências de pequeno porte contém em média 3,9 funcionários e as agências de médio porte têm em média 33,9 funcionários. Em 2013, a maioria das agências analisadas (50,7\%) investiram em média 5\% ou mais do faturamento anual em ações de marketing/vendas, sendo que as agências de pequeno porte investiram $4,0 \%$ e as agências de médio porte investiram 3,8\%.

O presente estudo buscou levantar as estratégias de marketing externo, interno e interativo das agências de viagens/turismo pesquisadas. A Figura 1 apresenta os resultados de estratégias de marketing externo - por total e porte de agência (somatório dos níveis de escala 4 e 5). Os resultados do total das 109 agências pesquisadas indicam que a grande maioria (4/5 delas) utiliza com elevada frequência as seguintes estratégias: satisfação dos clientes, relacionamento com fornecedores/parceiros comerciais, oferta de garantias de serviços, gestão do relacionamento/lealdade dos clientes, marketing direto (contato pessoal, telefone ou mala direta) e conhecimento de mercado/concorrência. No entanto, menos da metade das agências de viagens/turismo pesquisadas assinalou investir em propaganda/imagem da marca como estratégia de marketing externo. 
Figura 1 - Estratégias de marketing externo - por porte de agência

\begin{tabular}{|c|c|c|c|}
\hline Satisfação dos clientes $(\sigma=0,8 ; x=4,4)$ & 92 & 95 & 93 \\
\hline Relacionamento fornecedores/parceiros $(\sigma=0,9 ; x=4,3)$ & 86 & 84 & 85 \\
\hline Garantias de serviços $(\sigma=1,1 ; x=4,1)$ & 81 & 68 & 79 \\
\hline Relacionamento/lealdade clientes $(\sigma=0,9 ; x=4,2)$ & 77 & 89 & 79 \\
\hline Marketing direto $(\sigma=1,1 ; x=4,1)$ & 70 & 89 & 73 \\
\hline Conhecimento mercado/concorrência $(\sigma=1,1 ; x=3,9)$ & 69 & 89 & 72 \\
\hline Focalização em clientes/mercados $(\sigma=1,1 ; x=3,8)$ & 63 & 68 & 64 \\
\hline Serviço diferenciado/inovador $(\sigma=1,3 ; x=3,6)$ & 60 & 68 & 61 \\
\hline Inovação na prospecção $(\sigma=1,2 ; x=3,5)$ & 53 & 74 & 57 \\
\hline Precificação serviços $(\sigma=1,4 ; x=3,4)$ & 49 & 79 & 54 \\
\hline Promoção de vendas $(\sigma=1,3 ; x=3,6)$ & 49 & 79 & 54 \\
\hline Propaganda da marca $(\sigma=1,3 ; x=3,4)$ & 42 & 74 & 48 \\
\hline \% Agência pequena $(n=90)$ & \% Agência média ( $n=19$ ) & \% Total $(n=109)$ & \\
\hline
\end{tabular}

Fonte: Agências de viagens e turismo de Santa Catarina pesquisadas(2014)

Em termos de média aritmética, os resultados apresentam tendência central de utilização semelhante às apontadas na análise de frequência apresentada, tendo exatamente médias mais elevadas aquelas estratégias com maior frequência de utilização. Cabe destacar que cinco das variáveis mensuradas apresentam desvio padrão ligeiramente elevado (superior à $20 \%$ da escala utilizada), o que indica possível tendência de dispersão dos dados em relação a média e possível presença de agrupamentos (clusters) entre as agências pesquisadas. As estratégias com desvio padrão mais elevado são: precificação dos serviços, oferta de serviços diferenciados/inovadores, ofertas de promoção de vendas, propaganda/imagem da marca e inovação na prospecção de clientes.

Quanto ao porte da organização, os resultados apresentam diferença na frequência de aplicação entre as agências de pequeno e médio portes. As agências de porte médio utilizavam com maior frequência grande parte das estratégias mensuradas, principalmente: propaganda/imagem da marca, oferta de promoção de vendas, precificação de serviços, inovação na prospecção de clientes, conhecimento de mercado/concorrência, marketing direto, relacionamento/lealdade dos clientes, oferta de serviços diferenciados/inovadores e focalização em determinados clientes/mercados. No entanto, os resultados do teste t-Student revelaram, ao nível de significância de 5\%, que há diferença estatística significativa na prática de marketing externo das agências pesquisadas apenas nas estratégias de propaganda/imagem da marca $(p=0,020)$, promoção de vendas $(p=0,040)$, marketing direto $(p=0,027)$ e precificação dos serviços $(p=0,016)$.

A Figura 2 mostra os resultados das estratégias de marketing interno e interativo aplicadas - por total e porte de agência (somatório dos níveis de escala 4 e 5). Os resultados das 109 agências pesquisadas apontam que a grande maioria ( $4 / 5$ delas) utiliza com frequência elevada as se- 
guintes estratégias: remuneração/incentivo de funcionários, atendimento às reclamações/serviços de pós-venda, forma de atendimento/interação com clientes, comunicação/interação interna, qualidade/produtividade dos serviços, perfil dos funcionários (aparência e qualificação), satisfação/retenção de funcionários e aspectos do cenário de serviços, que inclui a ambientação da loja.

Figura 2 - Estratégias de marketing interno e interativo - por porte de agência

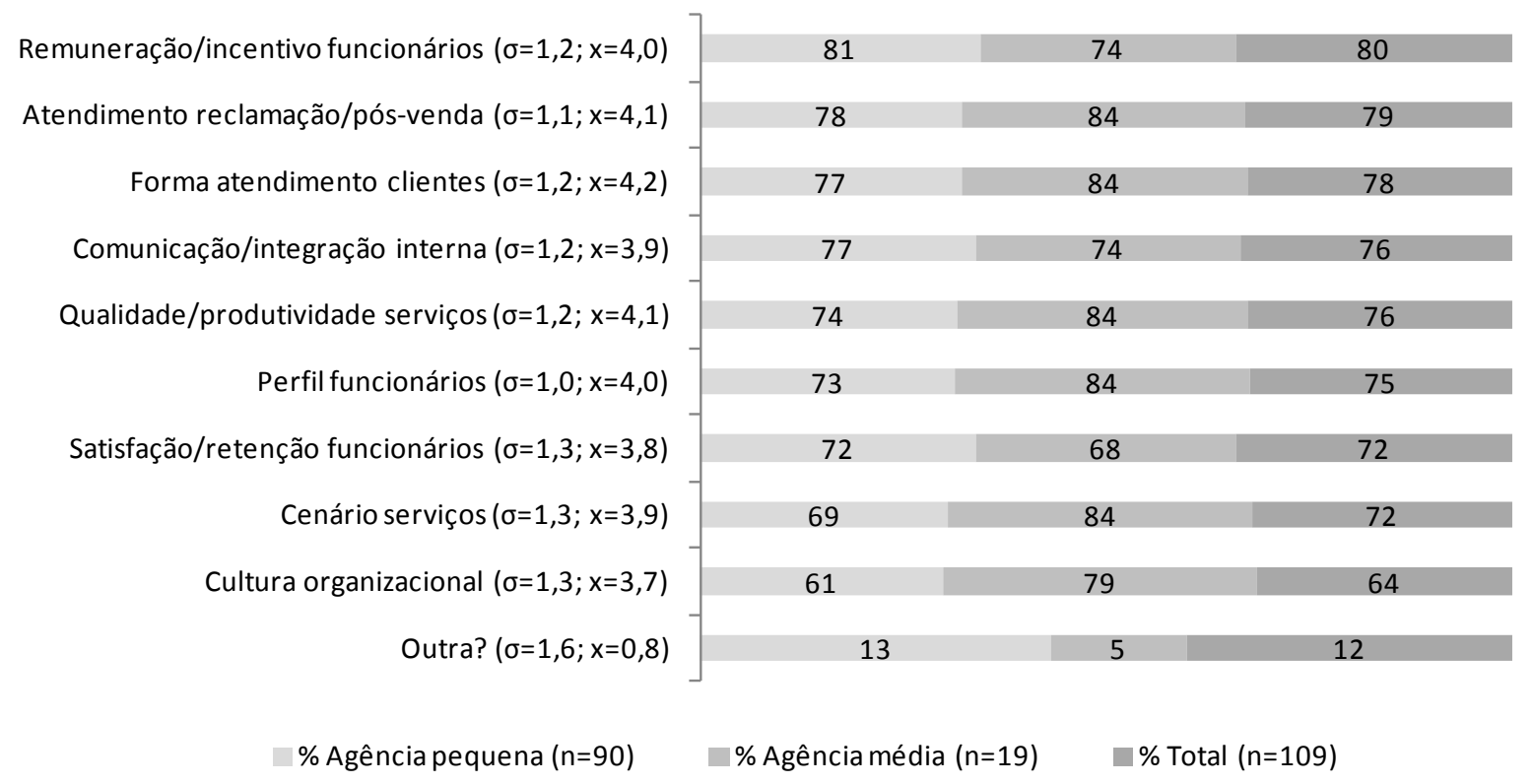

Fonte: Agências de viagens e turismo de Santa Catarina pesquisadas (2014)

Em termos de média aritmética, os resultados indicaram que a tendência central de utilização das estratégias mensuradas ocorre também em nível semelhante às apontadas na análise de frequência apresentada, principalmente as seguintes estratégias: perfil dos funcionários, qualidade/produtividade dos serviços, forma de atendimento/interação com clientes, atendimento às reclamações e remuneração/incentivo de funcionários. No entanto, a grande maioria das estratégias mensuradas apresenta desvio padrão ligeiramente elevado, o que sugere possível tendência de dispersão dos dados coletados e possível presença de clusters entre as agências pesquisadas.

Quanto ao porte da organização, os resultados apresentaram também diferença na frequência de aplicação entre as agências de pequeno e médio portes. As agências de porte médio utilizavam com maior frequência grande parte das estratégias de marketing mensuradas, principalmente: cultura organizacional, aspectos do cenário de serviços, perfil dos funcionários e qualidade/produtividade dos serviços. No entanto, os resultados do teste t-Student revelaram, ao nível de significância de $5 \%$, que não há diferença estatística significativa na prática de marketing interno e interativo das agências de pequeno e médio portes para qualquer das estratégias de mensuradas.

Outro objetivo específico do presente estudo foi analisar as estratégias de gestão do relacionamento com clientes. A Figura 3 apresenta os resultados de estratégias de gestão do relacionamento com clientes das agências pesquisadas - por total e porte de agência (somatório dos ní- 
veis de escala 4 e 5). Os resultados do total de agências pesquisadas apontaram que a grande maioria utiliza com elevada frequência as seguintes estratégias: oferecer atendimento cordial/prestativo, preocupação com a satisfação dos clientes, preocupação em atender as necessidades dos clientes, oferecer atendimento diferenciado/personalizado, solucionar conflitos/reclamações dos clientes, construir relacionamentos de longo prazo com clientes, dispor de pessoal de serviços competente/confiável, ter conhecimento sobre clientes-alvo e facilitar o acesso dos clientes aos serviços.

Figura 3 - Estratégias de relacionamento com clientes - por porte de agência

\begin{tabular}{|c|c|c|c|}
\hline Ter atendimento cordial/prestativo $(\sigma=0,5 ; x=4,7)$ & 98 & 100 & 98 \\
\hline Focalizar na satisfação dos clientes $(\sigma=0,5 ; x=4,6)$ & 97 & 100 & 97 \\
\hline Atender as necessidade dos clientes $(\sigma=0,5 ; x=4,7)$ & 96 & 100 & 96 \\
\hline Oferecer atendimento personalizado $(\sigma=0,8 ; x=4,5)$ & 91 & 100 & 93 \\
\hline Solucionar conflitos/reclamações $(\sigma=0,7 ; x=4,5)$ & 90 & 100 & 92 \\
\hline Construir relacionamentos longo prazo $(\sigma=0,7 ; x=4,5)$ & 91 & 95 & 92 \\
\hline Ter pessoal competente/confiável $(\sigma=0,7 ; x=4,6)$ & 90 & 95 & 91 \\
\hline Conhecer os clientes-alvo $(\sigma=0,8 ; x=4,2)$ & 84 & 100 & 87 \\
\hline Facilitar acesso/prontidão aos clientes $(\sigma=0,8 ; x=4,3)$ & 82 & 100 & 85 \\
\hline Executar ações de pós-venda $(\sigma=1,0 ; x=4,1)$ & 77 & 84 & 78 \\
\hline Oferecer novidades/promoções ( $\sigma=1,2 ; x=3,9)$ & 69 & 89 & 72 \\
\hline \% Agência pequena $(n=90)$ & $\% A$ & & \\
\hline
\end{tabular}

Fonte: Agências de viagens e turismo de Santa Catarina pesquisadas (2014)

Em termos de média aritmética, exceto a estratégia "oferecer novidades/promoções periódicas", todas as demais estratégias apresentaram resultados de média elevado (superior a 4), confirmando os resultados da análise de frequência apresentados. Além disso, quase a totalidade das estratégias mensuradas apresentou desvio padrão baixo, indicando baixa dispersão dos valores em relação à média e maior confiabilidade dos dados coletados.

Com relação ao porte da organização, os resultados apontaram que as agências de médio porte utilizam com mais frequência todas as estratégias de marketing de relacionamento com clientes mensuradas. A totalidade das médias agências utilizam com mais frequência as seguintes estratégias: facilitar o acesso dos clientes aos serviços, dispor de conhecimento sobre clientesalvo, solucionar conflitos/reclamações dos clientes, oferecer atendimento diferenciado/personalizado, atentar para as necessidades dos clientes, atentar para a sua satisfação e oferecer-Ihes atendimento cordial/prestativo. No entanto, os resultados do teste t-Student revelaram, em nível de significância de 5\%, que há diferença estatística significativa na prática de marketing 
relacionamento das agências pesquisadas apenas nas estratégias de esforço para construir relacionamento de longo prazo com clientes $(p=0,019)$ e oferecer facilidades de acesso/prontidão aos clientes $(p=0,045)$.

A Figura 4 apresenta os resultados das estratégias de marketing que mais contribuem para vantagem competitiva, segundo a opinião das agências de viagens/turismo pesquisadas. As estratégias de marketing apontadas pela maioria das agências pesquisadas são: qualidade/produtividade dos serviços, atendimento/interação com clientes, satisfação dos clientes, relacionamento/lealdade dos clientes, conhecimento de mercado/concorrência, serviços de pósvenda, marketing pela Web/Internet, gestão de fornecedores/parceiros de serviços, perfil dos funcionários, serviço diferenciado/inovador, comunicação/integração interna, remuneração/incentivo de funcionários, oferta de promoção de vendas e comunicação/ e imagem da marca. As estratégias de marketing menos assinaladas são: precificação dos serviços, focalização em determinados clientes/mercados, inovação na prospecção de clientes e cenários/ambiente de serviços.

Figura 4 - Estratégias que mais contribuem para vantagem competitiva - por porte de agência

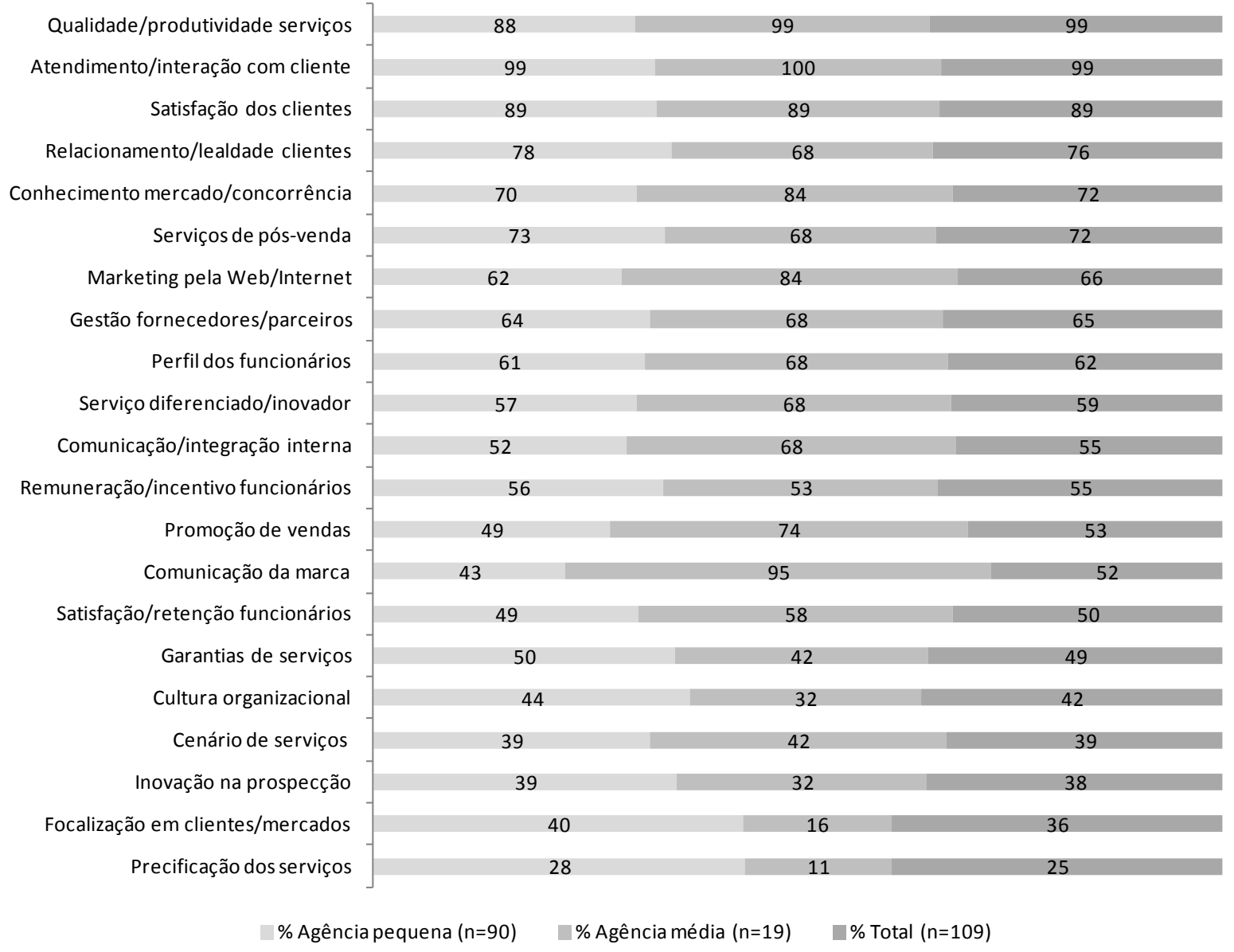

Fonte: Agências de viagens e turismo de Santa Catarina pesquisadas (2014)

No caso das pequenas agências, as estratégias de marketing mais assinaladas são: atendimento/interação com clientes, satisfação dos clientes, qualidade/produtividade dos serviços, rela- 
cionamento/lealdade dos clientes, serviços de pós-venda e conhecimento de mercado/concorrência. No caso das médias agências, as estratégias de marketing mais assinaladas foram: atendimento/interação com clientes, qualidade/produtividade dos serviços, comunicação/imagem da marca, satisfação dos clientes, conhecimento de mercado/concorrência, marketing na Web/Internet, oferta de promoção de vendas, relacionamento/lealdade dos clientes, serviços de pós-venda, gestão de fornecedores/parceiros de serviços, perfil dos funcionários, serviço diferenciado/inovador e comunicação/integração interna. Entretanto, os resultados do teste tStudent revelaram, ao nível de significância de $5 \%$, que não há diferença estatística significativa na opinião das agências pesquisadas sobre o assunto.

\subsection{Discussão}

A análise dos resultados do estudo revela que as agências de viagens/turismo pesquisadas utilizam estratégias de marketing externo, interno/interativo e de relacionamento com os clientes, tanto as agências de médio porte como de pequeno porte. A grande maioria delas utiliza, com elevada frequência, as seguintes estratégias: satisfação dos clientes, relacionamento com fornecedores/parceiros, oferta de garantias de serviços, oferta de serviços diferenciado/inovador, relacionamento com clientes, marketing direto, conhecimento de mercado/concorrência, remuneração/incentivo de funcionários, atendimento às reclamações/pós-venda, atendimento/interação com clientes, comunicação interna, qualidade dos serviços, perfil dos funcionários, satisfação de funcionários, cenário de serviços, oferecer atendimento diferenciado/personalizado, solucionar conflitos/reclamações dos clientes, dispor de pessoal competente/confiável, conhecer os clientes e facilitar o acesso aos serviços da agência.

O resultado do estudo que aponta o uso de serviços diferenciados/inovação como estratégia de marketing corroboram com as afirmações de Kotler, Bowen e Makens (2006) e Da Silva Flores, De Sena Cavalcante e Raye (2012), segundo os quais estratégias utilizadas no setor incluem inovações em produtos, processos e no relacionamento com clientes. Os resultados sobre estratégias de marketing mais utilizadas corroboram também com o modelo teórico proposto por Viljoen, Roberts-Lombard e Jooste (2015), o qual apresenta como estratégias de marketing para agências de viagens/turismo, que podem contribuir para a redução da desintermediação e para a retenção dos clientes, aspectos relacionados à interação pessoal, serviço diferenciado, conhecimento do produto e oferecer serviço de qualidade.

Os resultados do estudo apontaram também que a grande maioria das agências de viagens/turismo pesquisadas investe, com elevada frequência, no relacionamentos com fornecedores/parceiros comerciais do setor de turismo, seja como estratégia de marketing ou como forma de obter apoio/cooperação na implementação das estratégias (algo assinalado por $84 \%$ das agências de médio porte e por $86 \%$ das agências de pequeno porte). Isso confirma os achados do estudo de Hoffmann et al. (2015), que apontou como estratégia de marketing do setor desenvolver relações com outras organizações da cadeia produtiva do turismo. Esse resultado confirma também a proposição de Kavoura et al. (2013), segundo o qual o setor de serviços precisa obter a co- 
operação de outros agentes de mercado para otimizar a implementação da estratégia de marketing da organização.

Cid et al. (2015) propuseram que as agências de viagens/turismo estão utilizando estratégias contemporâneas de marketing, baseadas no uso da tecnologia da informação e comunicação, especialmente redes sociais e mídias sociais. Os resultados do presente estudo apontaram que o marketing direto (incluindo o uso de mala direta, e-mail marketing e mídias sociais) é bastante utilizado pela grande maioria das agências de viagens/turismo pesquisadas (89\% das agências de médio porte e $70 \%$ das agências de pequeno porte). Esse resultado confirma também os achados de Da Silva Flores, De Sena Cavalcante e Raye (2012), que revelaram o uso da tecnologia da informação/comunicação como estratégia de marketing, principalmente no relacionamento com clientes e fornecedores/parceiros comerciais, e na comercialização dos serviços. De fato, como apontado por Radu et al. (2014), com o advento da Internet, as agências de viagens/turismo precisaram adaptar as suas estratégias de marketing e intensificar o uso de mídia social, aplicativos móveis, marketing de relacionamento e serviços diferenciados. Outros exemplos de estratégias contemporâneas utilizadas pela maioria das agências pesquisadas são: investir em inovação na prospecção de novos clientes ( $74 \%$ das agências de médio porte e $53 \%$ das agências de pequeno porte) e oferecer serviço diferenciado/inovação ( $68 \%$ das agências de médio porte e $60 \%$ das agências de pequeno porte).

Corroborando com Moyano et al. (2015), que investigou o desenvolvimento de estratégias de marketing baseadas na satisfação do consumidor, os resultados do presente estudo apontam ainda que a grande maioria das agências de viagens/turismo pesquisadas adota como estratégia de marketing investir na satisfação dos clientes ( $97 \%$ delas), no atendimento das necessidades dos clientes-alvo (96\%) e na construção de relacionamentos de longo prazo com os clientes (92\%). Isso confirmam as proposições de Sampaio e Fagundes (2008) e Dwyer e Schurr (2006), segundo os quais uma premissa básica do marketing nos serviços de turismo é focar na satisfação dos clientes e na construção de relacionamentos de longo prazo com os clientes-alvo.

Barboza et al. (2013) alertaram para a importância da inovação em organizações que atuam em mercados de concorrência acirrada, com variedade de ofertas e elevado nível de exigência dos consumidores. O presente estudo apontou que a maioria das agências de viagens/turismo pesquisadas investe na introdução de serviço diferenciado/inovação como estratégia de marketing (68\% das agências de médio porte e $60 \%$ das agências de pequeno porte), e que esta estratégia foi apontada como uma das que mais contribui para a vantagem competitiva no setor (68\% das agências de médio porte e $57 \%$ das agências de pequeno porte). Isso confirma também os achados de Freire (2005) no ramo de hotelaria e de Maske (2012) no setor de turismo, segundo os quais gestores de turismo estão procurando ativamente introduzir inovações na organização e percebem que a inovação tem hoje papel fundamental no desempenho da organização, seguido da orientação empreendedora e da orientação para o mercado. No entanto, diferente dos achados de Barboza et al. (2013), os resultados do presente estudo apontaram que a precificação dos serviços 
não é percebida pela maioria como uma estratégia de marketing que contribui para obtenção de vantagem competitiva no setor.

\section{CONSIDERAÇÕES FINAIS}

Este estudo analisou as estratégias de marketing aplicadas pelas organizações de serviços do setor de agências de viagens/turismo do Estado de Santa Catarina e, para isso, 109 organizações foram investigadas sobre as estratégias de marketing externo, interno, interativo e de gestão do relacionamento com clientes aplicadas; e sobre as estratégias de marketing que mais contribuem para a vantagem competitiva no setor de agências de viagens/turismo.

Os resultados apontaram que a grande maioria das agências pesquisadas utiliza as seguintes estratégias de marketing: foco na satisfação dos clientes, construção de relacionamentos com fornecedores/parceiros de serviços, oferta de garantias de serviços, relacionamento/lealdade dos clientes, marketing direto, obtenção de conhecimento sobre mercado/concorrência (marketing externo); remuneração/incentivo de funcionários, comunicação/interação interna, investe no perfil dos funcionários e na satisfação/retenção de funcionários (marketing interno); qualidade/produtividade dos serviços, forma de atendimento/interação com clientes, execução de serviços de pós-venda e aspectos do cenário de serviços (marketing interativo). No relacionamento com clientes, a grande maioria das agências pesquisadas utiliza as seguintes estratégias de marketing: oferecer atendimento cordial/prestativo, zelar pela satisfação no relacionamento, atentar as necessidades dos clientes, oferecer atendimento personalizado, solucionar conflitos/reclamações dos clientes, construir relacionamentos de longo prazo, dispor de pessoal competente/confiável, ter conhecimento sobre clientes-alvo e facilitar o acesso dos clientes aos serviços.

Quanto ao porte das organizações, os resultados revelaram que as agências de porte médio utilizam com maior frequência a maioria das estratégias de marketing mensuradas, principalmente: propaganda/imagem da marca, ofertas de promoção de vendas, precificação de serviços, inovação na prospecção de clientes, dispor de conhecimento sobre mercado/concorrência, marketing direto, relacionamento/lealdade dos clientes, oferta de serviços diferenciados/inovadores, focalização em determinados clientes/mercados, cultura organizacional, aspectos do cenário de serviços, perfil dos funcionários e qualidade/produtividade dos serviços oferecidos. Os resultados do teste t-Student revelaram que há diferença estatística significativa na prática de marketing externo das agências pesquisadas nas estratégias de propaganda/imagem da marca, promoção de vendas, marketing direto e precificação dos serviços. E na prática de marketing relacionamento com clientes nas estratégias de esforço para construir relacionamento de longo prazo com clientes e oferecer facilidades de acesso/prontidão aos clientes.

Com relação à vantagem competitiva no setor, na opinião da maioria das agências pesquisadas as estratégias de marketing que mais contribuem são: qualidade/produtividade dos serviços, atendimento/interação com clientes, satisfação dos clientes, relacionamento/lealdade dos clientes, conhecimento de mercado/concorrência, serviços de pós-venda, marketing pela Web/Internet, gestão de fornecedores/parceiros de serviços, perfil dos funcionários, serviço dife- 
renciado/inovador, comunicação/integração interna, remuneração/incentivo de funcionários, oferta de promoção de vendas e comunicação/imagem da marca. Os resultados do teste t-Student revelaram, no entanto, não há diferença estatística significativa na opinião das agências pesquisadas sobre o assunto.

O estudo tem contribuição teórica relevante. Ele possibilitou a ampliação do conhecimento acadêmico sobre as estratégias marketing aplicadas pelas agências de viagens/turismo, um ramo de atividade com carência de estudos acadêmicos como apontado por Barros (2005) e Borges e Guardia (2012). O trabalho contribui também para ampliar o conhecimento acadêmico sobre a aplicação de estratégias de marketing externo, interno, interativo em organizações de pequenos e médios portes, como recomendado por Sarquis (2009) e Sarquis e Ikeda (2009). O estudo contém também várias evidências empíricas sobre elementos de estratégias de marketing que podem auxiliar na realização de futuros estudos acadêmicos e na formulação de hipóteses de pesquisa. Confirmando o que foi apontado por Vasconcelos, Souza e Guimarães (2010), o estudo revelou que estratégias de marketing aplicadas pela maioria das organizações são aspectos de satisfação dos clientes e qualidade dos serviços, e que a maioria das agências pesquisadas investe na satisfação dos clientes, no relacionamento com clientes/fornecedores, na oferta de garantias de serviços e na oferta de serviços diferenciados e inovação. O estudo apontou também que a maioria das agências introduz inovação na prospecção de novos clientes/negócios, confirmando a proposição teórica de Barboza et al. (2013) que inovação no relacionamento com clientes é uma estratégia de marketing aplicada no setor de serviços. O estudo apontou que inovações na prospecção com clientes é um constructo teórico que deve ser considerado na análise das estratégias de marketing no setor de serviços de agências de viagens.

Embora os objetivos do estudo tenham sido atingidos e os cuidados metodológicos necessários tenham sido observados, algumas limitações do estudo devem ser destacadas. Como a população estudada é representada por amostragem não probabilística (seleção por conveniência), os resultados do estudo não são conclusivos e podem não refletir o conjunto das organizações do setor de agências de viagens/turismo do Estado de Santa Catarina. Além disso, a população-alvo é composta apenas pelas agências de viagens/turismo classificadas como emissivas e a amostra estudada contém apenas as organizações identificadas a partir de informações de dois fornecedores do setor.

Como direcionamento para futuros estudos no campo da estratégias de marketing para agências de viagens/turismo, propõe-se realizar pesquisa no Estado de Santa Catarina com tamanho amostral mais representativo e envolvendo processo de amostragem probabilístico, com a seleção de participantes pelo método aleatório; executar estudo qualitativo com agências de viagens/turismo do Estado de Santa Catarina (com entrevistas em profundidade ou focus group) para ampliar a compreensão sobre os resultados do presente estudo e obter explicações para as diferenças verificadas no desempenho competitivo em função do porte da organização; verificar diferenças na prática de estratégias de marketing entre as agências de micro e pequeno portes do Estado de Santa Catarina; investigar outras variáveis de pesquisa não consideradas no presente 
estudo, como aspectos do relacionamento com fornecedores, dificuldades enfrentadas e inovações introduzidas no processo de marketing pelas agências de viagens/turismo investigadas; estudar as estratégias de marketing que mais contribuem para vantagem competitiva em agências de viagens/turismo em outros estados do Brasil, e comparar os resultados com os dados obtidos no presente estudo.

\section{REFERÊNCIAS}

American Marketing Association. AMA Dictionary. Recuperado em 09.10.2014 de https://www.ama.org/resources/Pages/Dictionary.aspx?dLetter=M.

Barboza, M.; Ormay, C.; Arruda, E.; Toaldo, A.; Silva, D. (2013). Análise da gestão de marketing das escolas privadas através da operacionalização das estratégias do composto de marketing. Revista Brasileira de Administração Científica, 4 (1)

Barros, F. (2005). O alinhamento estratégico da utilização da internet em agências de viagens e turismo operando no brasil. Dissertação, Mestrado em Administração, Instituto COPPEAD de Administração, Universidade Federal do Rio de Janeiro, Rio de Janeiro, RJ, Brasil.

Beni, M. (2006). Política e planejamento de turismo no Brasil. São Paulo: Aleph.

Borges, A.; Guardia, M. (2012). As agências de viagens e turismo: um estudo das publicações em periódicos de turismo entre os anos 2006-2011. Revista Turismo: Estudos e Práticas, 1 (2).

Candioto, M. (2012). Agências de turismo no Brasil: embarque imediato pelo portão dos desafios. Rio de Janeiro: Elsevier.

Carvalho, J.; Hemais, M.; Motta, P. (2001). Do zen ao Techno: as tribos de Consumidores e a música nos cenários de serviços. Anais do XXV EnANPAD, Campinas, SP.

Cavalcanti, G. (2008) Imagem e Qualidade Percebida: A perspectiva dramatúrgica do marketing de serviços. Anais do III Encontro de Marketing da ANPAD, Curitiba, PR.

Cid, L., Brea, J., Valcarcel, D., \& Brea, J. (2015). Travel agencies under the influence of social networks on tourism. the case of Ourense. PASOS: Revista de Turismo y Patrimonio Cultural, 13(4, Special Issue), 829836.

Collis, J; Hussey, R. (2005). Pesquisa em administração: um guia prático para alunos de graduação e pósgraduação. Porto Alegre: Bookman.

Cronbach, L. (1951). Coefficient alpha and the internal structure of tests. Psychometrika, v.16, 297-334.

Da Silva Flores, L., De Sena Cavalcante, L., \& Raye, R. (2012). Marketing turístico: Estudo sobre o uso da tecnologia da informação e comunicação nas agências de viagens e turismo de Balneário Camboriú (SC, Brasil). Revista Brasileira de Pesquisa em Turismo, 6(3), 322-339.

Dwyer, F.; Schurr, P.; Oh, S. (2006). Desenvolvimento dos relacionamentos entre comprador e vendedor. RAE - Revista de Administração de Empresas - Clássicos, 46 (3), 111-136. 
Freire, S. (2005). Estratégias de marketing como ferramenta para a melhoria da gestão estratégica dos pequenos hotéis de Fortaleza. Dissertação de Mestrado Profissional em Administração da Universidade Federal do Ceará, Fortaleza, CE, Brasil.

Ghemawat, P.; Rivkin, J. (2006). Creating competitive advantage. Boston: Harvard Business School Note. Grabicoski, J. (2008). Estratégias de marketing para minimização do desequilíbrio entre demanda e a capacidade no setor de serviço no Brasil. Dissertação de Mestrado em Administração Estratégica da Pontifica Universidade Católica do Paraná, Curitiba, PR, Brasil.

Grönroos, C. (1984). Service quality model and its marketing implications. European Journal of Marketing, $18(4), 36-44$.

Hoffmann, V., Vieira, D., Junior, E., \& Melo, M. (2015). Estrategia empresarial en períodos de crisis. Estudios y Perspectivas en Turismo, 24, 663-682.

Hollensen, S. (2014). Marketing Management. A Relationship Approach. Pearson Higher Ed.

Huang, Y., \& Rundle-Thiele, S. (2014). The moderating effect of cultural congruence on the internal marketing practice and employee satisfaction relationship: An empirical examination of Australian and Taiwanese born tourism employees. Tourism Management, 42, 196-206.

Kavoura, A., Katsoni, V., Vassiliadis, C., \& Vlachopoulou, M. (2013). From e-business to c-commerce: collaboration and network creation for an e-marketing tourism strategy. In Tourismos, 8 (3), 113-128.

Kotler, P., Bowen, J., \& Makens, J. (2006). Marketing for hospitality and tourism. Upper Saddle River, NJ: Prentice hall.

Kotler, P., Keller, K., Ancarani, F., \& Costabile, M. (2014). Marketing management. Pearson.

Lovelock, C., Patterson, P., \& Wirtz, J. (2014). Services marketing. Pearson Australia.

Lucena, R. (2011). Análise do processo de formação de estratégias de marketing no Jornal Correio da Paraíba. Dissertação de Mestrado em Administração da Universidade Federal da Paraíba, João Pessoa, Paraíba, Brasil.

Machado, D.; Almeida, D.; Scarpin, J. (2011) Endividamento e lucratividade: um estudo em empresas familiares e não familiares que compõem o índice ibrx-100 da Bovespa. Anais do Congresso de Contabilidade $e$ Controladoria da USP, São Paulo, Brasil.

Maske, D. (2012). Relação entre orientação empreendedora, inovação, orientação para o mercado e desempenho em empresas turísticas. Dissertação de Mestrado em Turismo e Hotelaria da Univali - Universidade do Vale do Itajaí, Balneário Camboriú, SC, Brasil.

Ministério do Turismo. Estudo, pesquisas e dados sobre o setor de turismo. Recuperado em 28.02.14 de http://www.dadosefatos.turismo.gov.br/dadosefatos/estatisticas indicadores/estatisticas basicas turism of

Moyano, C., Lengler, J., Angnes, D., \& Sampaio, F. (2015). Estratégia para fidelização do cliente em turismo: O caso de uma Oktoberfest no Brasil. Revista Turismo - Visão e Ação, 17 (2) 387-413.

Murphy, K.; Davidshofer, C. (1988). Psychological testing: principles and applications. New Jersey: Prentice Hall. 
Navickas, V.; Malakauskaite, A. (2015). The possibilities for the identification and evaluation of tourism sector competitiveness factors. Engineering Economics, 61(1), 37-44.

Okada, S.; Souza, E. (2011). Estratégias de marketing digital na era da busca. REMark - Revista Brasileira de Marketing, 10 (1), 46-72.

Organização Mundial do Turismo (2010). E-bussiness para turismo. Porto Alegre: Bookman.

Pelissari, A.; Fassarella, L.; Gonzalez, I.; Duque, W.; Setubal, F. (2011). Estratégias de marketing utilizadas na obtenção de vantagem competitiva. Anais do VIII SEGeT - Simpósio de Excelência em Gestão e Tecnologia. Resende, RJ, Brasil.

Polo-Peña, A., Frías-Jamilena, D., \& Rodríguez-Molina, M. (2012). Marketing practices in the Spanish rural tourism sector and their contribution to business outcomes. Entrepreneurship \& Regional Development, 24(7-8), 503-521.

Queiroga, A. (2011). Processo de formação de estratégias de marketing na hotelaria paraibana. Dissertação de Mestrado em Administração da Universidade Federal da Paraíba, João Pessoa, Paraíba, Brasil.

Radu, A., Orzan, M., Macovei, O., \& Orzan, O. (2014). impact of online tourism marketing in modern business: an overview of Romania's market. International Journal of Economic Practices and Theories, 4(2), 318321.

Ribeiro, A.; Grisi, C.; Saliby, P. (1999). Marketing de relacionamento como fator-chave de sucesso no mercado de seguros. RAE - Revista de Administração de Empresas, 39 (1), 31-41.

Richardson, R. (2008). Pesquisa social: métodos e técnicas. São Paulo: Atlas.

Rocha, A.; Silva, J. (2006). Marketing de serviços: retrospectiva e tendências. RAE - Revista de Administração de Empresas. São Paulo, SP.

Rocha, T.; Jansen, C.; Lofti, E.; Fraga, R. (2013). Estudo exploratório sobre o uso das redes sociais na construção do relacionamento com clientes. Revista Brasileiro de Gestão de Negócios, 15 (47), 262-282.

Rodríguez, C., Martínez-Fernández, V., Juanatey-Boga, O., \& Fernández, M. (2014). El marketing de afiliación como herramienta de comunicación y gestión de las agencias de viaje en el mercado virtual: Estudio del caso español. Estudios y perspectivas en turismo, 23(1), 60-80.

Sampaio, D.; Fagundes, A. (2008). Estratégias de marketing: um estudo prático. PRETEXTO, 9 (2), 97-116.

Santa Maria, J. (2009). Estratégias de marketing de relacionamento para instituições de ensino superior privadas de Porto Alegre - RS. Tese de Doutorado em Engenharia de Produção da Universidade Federal de Santa Catarina, Florianópolis, SC, Brasil.

Santos, C.; Fernandes, D. (2008). A recuperação de serviços como ferramenta de relacionamento e seu impacto na confiança e lealdade dos clientes. RAE - Revista de Administração de Empresas, 48 (1), 10-24.

Sarquis, A. (2009). Estratégias de marketing para serviços: como as organizações serviços devem estabelecer e implementar estratégias de marketing. São Paulo: Atlas. 
Sarquis, A.; Ikeda, A. (2009). Segmentação de mercado no ramo de agências de comunicação. Revista de Gestão USP, 16 (2), 101-119.

Sarquis, A.; Pizinatto, N. (2013). Modelo de diagnóstico mercadológico no setor de serviços. Revista de Negócios, 8 (2), 81-100.

Saunders, M; Lewis, P.; Thornhill A. (2003). Research Methods for Business Students. (3a. ed.) Prentice Hall: Pearson Education.

Scarpin, M.; Mondini, L.; Neumann, M.; Machado, D. (2011). Desenvolvimento de um ambiente propício para inovação no setor de serviços: uma análise em empresas de serviços contábeis do Vale do Itajaí - SC. RCO - Revista De Contabilidade E Organizações, 5 (13), 91-108.

Shemwell, J.; Croni, J. (1994). Services marketing strategies for coping with demand/ supply imbalances. Journal of Services Marketing, 8, 14-24.

Standing, C.; Tang-Taye, J. \& Boyer, M. (2014). The impact of the Internet in travel and tourism: a research review 2001-2010. Journal of Travel \& Tourism Marketing, 31(1), 82-113.

Tomazzoni, E., \& Bock, I. (2013). Marketing e gestão estratégica de hotelaria: Um estudo bibliométrico. Revista Brasileira de Pesquisa em Turismo, 7(1), 35-50.

Vasconcelos, I.; Souza, J.; Magalhães, S. (2010). A satisfação da prestação de serviços de contabilidade: o caso da R.A. Contabilidade. Revista da Universidade Vale do Rio Verde, 8 (1).

Viljoen, K., Roberts-Lombard, M., \& Jooste, C. (2015). Reintermediation strategies for disintermediated travel agencies: a strategic marketing perspective. International Business \& Economics Research Journal, 14(3), 561-574.

Artigo recebido em: 18/02/2015. Artigo aprovado em: 20/08/2015. 